\title{
Subcomponent self-assembly: a quick way to novel metallogels
}

\author{
Hana Bunzen, ${ }^{*[a]}$ Nonappa, ${ }^{[b]}$ Elina Kalenius, ${ }^{[a]}$ Sami Hietala ${ }^{[c]}$ and Erkki Kolehmainen ${ }^{[a]}$
}

Subcomponent self-assembly, introduced by the Nitschke group ${ }^{[1]}$ is a process which allow complex structures to be generated from simple building blocks (generally aldehydes and amines). In this bottom-up approach, the building blocks spontaneously self-assemble around templates (usually metal ions) leading to a simultaneous covalent $(\mathrm{C}=\mathrm{N})$ and dative $(\mathrm{N}-$ metal) bonds formation. The method has been successfully used to construct well-defined metal-organic macrocycles, helicates, catenanes, rotaxanes, grids, ${ }^{[2]}$ and cages. ${ }^{[3]}$ Our field of interest lies not in building-up of defined structures but in designing gelator molecules for a formation of supramolecular gels as functional nanomaterials. Herein, we report on a facile and quick method which leads to an in situ preparation of supramolecular metallogels by applying the concept of subcomponent selfassembly. We found that by utilizing this approach, multistimuliresponsive metallogels become easily accessible and tunable.

Metallogels (or metal-organic gels) ${ }^{[4]}$ belong to the group of supramolecular gels, ${ }^{[5]}$ i.e. gels formed by self-assembly of small molecules, often called low-molecular-weight gelators (LMWGs), into a three-dimensional network with solvent molecules immobilized inside the structure. ${ }^{[5 \mathrm{~d}, 6]}$ In contrast to conventional supramolecular gels, these gels are formed by gelator molecules containing metal ions. This makes them very attractive materials regarding potential applications, because by incorporating metals into gels, properties such as luminescence, magnetism, catalytic and redox activity can be introduced. ${ }^{[4 a, 4 b]}$

In a conventional gel preparation, gelator molecules are synthesized beforehand and then dissolved in a suitable solvent usually upon heating. This process is time and resource demanding. Therefore, new and simple strategies in a gel preparation are needed. Recently, there were several reports on gels formed by molecules which were synthesized directly in a gelling solvent from suitable components. ${ }^{[7]}$ This approach of in situ gelator synthesis undoubtedly saves time and resources. The subcomponent self-assembly naturally is a good way to apply the in situ approach to metallogels. It offers indisputable advantages including (i) shortening synthesis, (ii) gelation at ambient temperature (if components are soluble in the gelling solvent), and most importantly (iii) easy accessibility of a wealth of gel systems by facile exchange of one of the reaction components (or more). In this article we focus on the fine-tunability of the gels by exchanging the metal ions.

The design of a gelator molecule for the subcomponent selfassembly is based on utilizing suitable imine bond precursors. To form strong metal-binding sites, the use of 2-pyridinecarboxaldehyde or its derivative is very convenient because the nitrogen atom of the pyridine unit together with the nitrogen atom from the newly formed imine group constitutes a strong bidentate ligand similar to 2,2 '-bipyridine. Therefore, the amine moiety is generally easier to modify and tune for our purpose because the above mentioned ligand will form with nearly every primary amine. Instead of using rigid di- or triamines like it is usually done in the construction of defined metal-organic compounds, ${ }^{[2,3]}$ we used a monoamine attached to a steroidal part responsible for forming non-covalent interactions between the gelator molecules resulting in the formation of a three-dimensional network. In this work commercially available 2-pyridinecarboxaldehyde (2), synthetically easily accessible steroidal amine (1), and three divalent metal ions, namely $\mathrm{Cu}(\mathrm{II}), \mathrm{Ni}(\mathrm{II})$, and $\mathrm{Zn}$ (II), were used as the reactants (Scheme 1). After mixing these components in a 3:3:1 ratio, a gel formation in higher alcohols, selected aromatic solvents and tetrachloromethane was observed (Table 1). Remarkably, the gelation occurred at ambient temperature without the need of a heating/cooling cycle otherwise commonly used in a gel preparation. Interestingly, the order of the components, in which they were added, was not crucial and in all cases the addition of the final component acted as a chemical stimulus triggering the gel formation (Scheme S1 in the Supporting information).

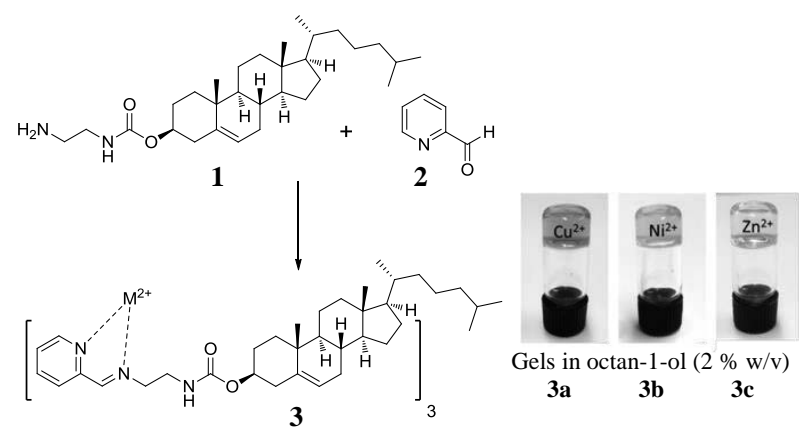

Scheme 1. Preparation of gelators 3a-c via subcomponent self-assembly.

We observed that the rate of the gel formation depends on the type of the used metal and decreases in the order $\mathrm{Zn}>\mathrm{Cu}>>\mathrm{Ni}$. The sol-to-gel transition was monitored in detail by time sweep rheological measurements (Fig. S12 in the Supporting information). Upon aggregation leading to gelation, there was a rapid increase in $\mathrm{G}^{\prime}$ and $\mathrm{G}^{\prime \prime}$ and instantaneous decrease in the phase angle to $\sim 20^{\circ}$. Based on the results, the gelation time was revealed as 15,18 and $55 \mathrm{~min}$ for gels of $\mathbf{3 c}, \mathbf{3 a}$ and $\mathbf{3 b}$, respectively, which is a relatively quick process. However, upon an ultrasonic treatment, the gel formation was much faster and the gelation time reduced to several minutes (10 $\min$ for $\mathbf{3 b}$ ) or even seconds (50 s for $\mathbf{3 c}$ and $90 \mathrm{~s}$ for 3a). Additionally, we investigated the rheological properties of the gels by frequency sweep and stress sweep experiments which revealed the predominant nature of the elastic modulus $\mathrm{G}^{\prime}$, which was found to be higher than loss modulus $\mathrm{G}^{\prime \prime}$, confirming the systems under investigation are viscoelastic solids (see the Supporting information for details). 
Table 1. Gelation tests of 3a-c $\left(2 \%\right.$ w/v).$^{[a, b]}$

\begin{tabular}{|c|c|c|c|c|c|c|}
\hline Solvent & 1 & 2 & $1+2$ & 3a & $3 \mathbf{b}$ & $3 c$ \\
\hline benzene & $\mathbf{p C G}_{(30)}$ & S & S & S & $\mathbf{p C G}_{(40)}$ & S \\
\hline toluene & $\mathbf{p C G}_{(30)}$ & S & S & S & $\mathbf{p C G}_{(40)}$ & S \\
\hline $\mathrm{CHCl}_{3}$ & S & S & S & S & S & S \\
\hline $\mathrm{CCl}_{4}$ & $\mathbf{p C G}_{(50)}$ & S & S & S & $\mathbf{p C G}_{(25)}$ & $\mathbf{p C G}_{(30)}$ \\
\hline methanol & S & S & $\mathrm{P}$ & $\mathrm{P}$ & $\mathrm{P}$ & $\mathrm{P}$ \\
\hline butan-1-ol & S & S & S & $P$ & $\mathrm{P}$ & $\mathrm{P}$ \\
\hline pentan-1-ol & S & S & S & $\mathbf{O G}_{(15)}$ & $\mathbf{C G}_{(5.0)}$ & $\mathbf{O G}_{(15)}$ \\
\hline hexan-1-ol & S & S & S & $\mathbf{O G}_{(10)}$ & $\mathbf{C G}_{(2.5)}$ & $\mathbf{O G}_{(10)}$ \\
\hline heptan-1-ol & S & S & S & $\mathbf{C G}_{(7.5)}$ & $\mathbf{C G}_{(2.5)}$ & $\mathbf{C G}_{(10)}$ \\
\hline octan-1-ol & S & S & S & $\mathbf{C G}_{(5.0)}$ & $\mathbf{C G}_{(0.7)}$ & $\mathbf{C G}_{(7.5)}$ \\
\hline water & I & S & I & I & I & I \\
\hline DMF & $\mathrm{P}$ & S & S & S & S & S \\
\hline DMSO & $\mathbf{O G}_{(15)}$ & S & $\mathrm{P}$ & S & S & S \\
\hline
\end{tabular}

[a] $\mathrm{S}=$ solution, $\mathrm{P}=$ precipitate upon cooling, $\mathrm{CG}=$ clear transparent gel, $\mathrm{OG}=$ opaque gel, $\mathrm{pG}=$ partial gel, $\mathrm{I}=$ insoluble at the solvent boiling point. [b] Values in parentheses denote a minimum gelation concentration (MGC, g/L).

We observed that visual, mechanical and thermal stability properties of the metallogels were dependent on both the solvent and the metal ions used. The higher the alcohol, the stronger and thermally more stable gels were formed (Fig. S2 in the Supporting information). Whereas the $\mathrm{Cu}(\mathrm{II})$ and $\mathrm{Zn}$ (II)-gels obtained from pentan-1-ol and hexan-1-ol were opaque, the gels formed in heptan-1-ol and octan-1-ol, and the Ni(II)-gels were transparent (Fig. S1 in the Supporting information). The Cu(II)-complex (3a) forms green gels with similar properties (meaning gel-to-sol transition temperature $T_{g}$ and minimum gelation concentration MGC) to that of the $\mathrm{Zn}$ (II)-complex (3c). In comparison, the $\mathrm{Cu}(\mathrm{II})$-gels formed in octan-1-ol (with MGC of $0.50 \% \mathrm{w} / \mathrm{v}$ ) are more stable in lower concentrations, whereas the $\mathrm{Zn}$ (II)-gels (with MGC of $0.75 \% \mathrm{w} / \mathrm{v}$ ) are more stable at higher concentrations (Fig. 1 and Fig. S3 in the Supporting information), although, the differences are not too significant. Contrarily, the gels formed by the $\mathrm{Ni}(\mathrm{II})$-complex (3b) in higher alcohols are more thermally stable compared to the other two systems. For instance, the gel-sol phase transition temperature of its $2 \% \mathrm{w} / \mathrm{v}$ gels in higher alcohols is higher than $95{ }^{\circ} \mathrm{C}$ (Fig. S2 in the Supporting information). Moreover, the $\mathrm{Ni}(\mathrm{II})$-complex was found to be an excellent gelator for octan-1-ol with MGC of $0.07 \%$ w/v (i.e. $0.7 \mathrm{mg}$ in 1 $\mathrm{mL}$ ), which makes the system one of the best metal-organic gels ever reported (regarding MGC).

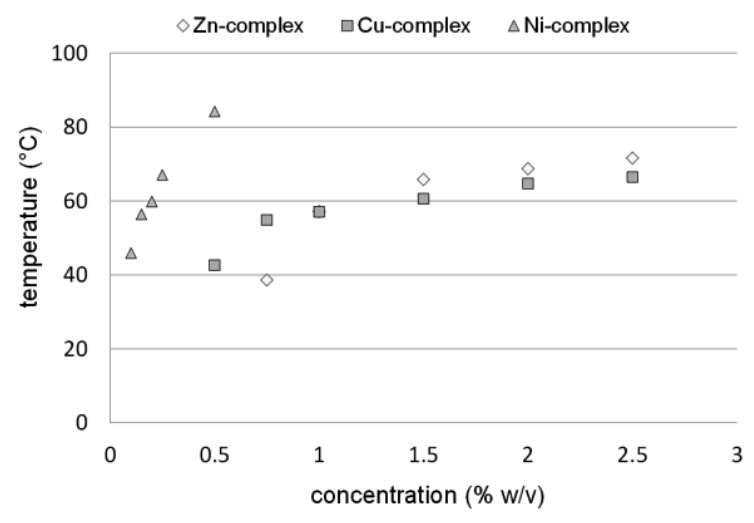

Figure 1. Phase diagram of gels of 3a-c in octan-1-ol.

In order to study the morphology of the metallogels, transmission electron microscopy (TEM) measurements were carried out for the octan-1-ol gels. A careful analysis of TEM micrographs (Fig. 2, and Fig. S17 in the Supporting information) revealed a highly entangled three-dimensional fibrillar network of xerogels of $\mathbf{3 b}$ and $\mathbf{3 c}$. However, electron micrographs obtained from 3a showed aggregated bundles of spherulite like structures interconnected to each other. Interestingly, an unusual formation of metal nanoparticles (for $\mathbf{3 b}$ and $\mathbf{3 c}$ ) was observed when a mixture of components was heated during the gel preparation. These preliminary results, supported by previous reports, ${ }^{[8]}$ indicate that under heating and aging of metallogels, an in situ generation of metal particles can take place.
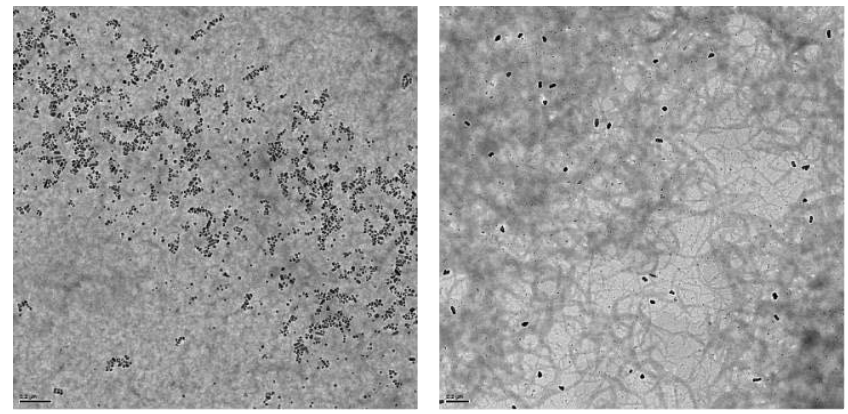

Figure 2. TEM micrographs of $1 \% \mathrm{w} / \mathrm{v}$ gel of $\mathbf{3 b}$ (right) and of ten times diluted $1 \% \mathrm{w} / \mathrm{v}$ gel of $\mathbf{3 b}$ (left) revealing a nanoparticle formation; bar indicates $0.2 \mu \mathrm{m}$.

The gelators were designed as 3:1 complexes (ligand-metal ratio) and the formation of these gelator molecules was confirmed by mass spectrometry measurements (Fig. S14-S16 in the Supporting information). Interestingly, an effective gelation occurred only when we used the ligand-metal ratio of $3: 1$, whereas, higher amounts of metal used did not lead to a gel formation (Fig. S4 in the Supporting information). The systems of different ligand-metal ratios, namely $3: 1,2: 1$ and $1: 1$, were studied by mass spectrometry measurements (Fig. S13-S16 in the Supporting information). The mass spectra measured from dissolved gel samples clearly show the abundance of the 3:1 metal complexes decreasing as the ligand to metal ratio (and gelling ability) is reduced, and revealed a formation of non-gelling metal complexes 2:1 and 1:1 instead. Additionally, it was observed that after an addition of excess amount of a metal salt caused a gel-tosol transition. However, the formation of the non-gelling 
complexes is a dynamic process and the gel could be restored by an addition of $\mathbf{1}$ and $\mathbf{2}$ in such amounts to achieve the 3:1 ligandmetal ratio again (Scheme 2$)$.

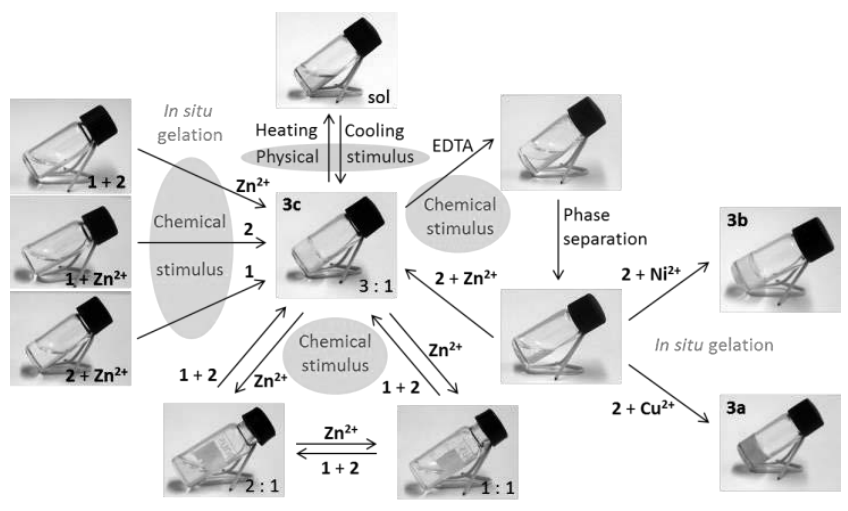

Scheme 2. Response of $3 \mathbf{c}$ in octan-1-ol (1\% w/v) to chemical and physical stimuli. Analogous results were observed when response to stimuli of 3a and $\mathbf{3 b}$ systems was studied (see Scheme S2 and S3 in the Supporting information)

As demonstrated, the gel behavior is strongly dependent on the metal used. Therefore, by choosing a suitable metal ion, gel properties can be fine-tuned and designed. Moreover, we are not limited only by the selection of available metals, but combinations of metal ions can be used as well. In our particular case various combinations of metal ions in different ratios could be used as long as the overall ligand-metal ratio stays $3: 1$. For instance, we prepared and studied mixed metallogels in a ligand-metal(1)metal(2) ratio of 3:0.5:0.5 (Fig. S5 and S6 in the Supporting information). By simple mixing different types of metal ions in certain ratios, gels with desired properties (e.g. a gel with a given value of $\mathrm{T}_{\mathrm{g}}$ ) are quickly accessible.

One of the reasons why supramolecular gels have gained so much attention over the last few years is that they offer the possibility to prepare materials responsive to various stimuli (such as temperature, light, ions, molecules, etc. $)^{[9]}$ by introducing a suitable switching or responsive functionality at a molecular level. In this work we succeed to prepare multi-responsive gels. Except the common gel-sol transition caused by an increase in temperature, also other responses of the systems were observed; including the described response to an excess of metal ions and the in situ gelation trigged by the final added component. Since all the three components are needed for an effective gelation, it can be expected that after removing one of the components a gel-sol transition should occur. Chemical stimulus like an addition of a strong chelating agent such as EDTA to a gel, indeed, induced a gel-sol transition. Expectably, the gel could be restored after a phase separation and an addition of a new portion of metal salt, and an addition of aldehyde $\mathbf{2}$ which was washed out to the aqueous layer (Scheme 2, and Scheme S2 and S3 in the Supporting information). Moreover, the added metal salt could be altered or even a mixture of metal salts could be used. This offers a unique possibility of switching from one gelator to the other with regard to the wanted properties.

In conclusion, the preparation of metallogels via subcomponent self-assembly, introduced in this article, was found to be powerful way to rapidly generated gels with defined properties. Additionally, these gels are multi-responsive: besides the common thermal stimulus, the gels are also sensitive to stoichiometry and chemical stimuli. Furthermore, the gels can be prepared on the minute down to the second scale at ambient temperatures which is exceptional and convenient with regards to potential applications. Finally, some of the gelators are supergelators outmatching most other known gelators. All these reasons make us eager to look into the wealth of properties and possibilities still to be found by modifying the amine and aldehyde components of the system. We believe that the subcomponent self-assembly strategy introduced in this work has a potential to become a general design route towards new smart gel materials.

\section{Acknowledgements}

Financial support by the Finnish Ministry of Education (National Doctoral Program of Organic Chemistry and Chemical Biology) is gratefully acknowledged (H.B.).

\section{References}

[1] a) V. E. Campbell, J. R. Nitschke, Synlett 2008, 20, 3077-3090; b) J. R. Nitschke, Acc. Chem. Res. 2007, 40, 103-112; c) M. Hutin, C. A. Schalley, G. Bernardinelli, J. R. Nitschke, Chem. Eur. J. 2006, 12, 4069-4076.

[2] C. D. Meyer, C. S. Joiner, J. F. Stoddart, Chem. Soc. Rev. 2007, 36, $1705-$ 1723 .

[3] T. K. Ronson, S. Zarra, S. P. Black, J. R. Nitschke, Chem. Commun. 2013, 49 , 2476-2490.

[4] a) A. Y. Tam, V. W. Yam, Chem. Soc. Rev. 2013, 42, 1540-1567; b) M. M. Piepenbrock, G. O. Lloyd, N. Clarke, J. W. Steed, Chem. Rev. 2010, 110, 1960-2004; c) F. Fages, Angew. Chem. Int. Ed. 2006, 45, 1680-1682.

[5] a) D. K. Smith, in Supramolecular Chemistry: From Molecules to Nanomaterials, Wiley\&Sons Ltd., Chichester, UK, 2012, pp. 3355-3376; b) J. W. Steed, Chem. Commun. 2011, 47, 1379-1383; c) P. Dastidar, Chem. Soc. Rev. 2008, 37, 2699-2715; d) R. G. Weiss, P. Terech, in Molecular Gels: Materials with Self-Assembled Fibrillar Networks, Springer, Netherlands, 2006; e L. A. Estroff, A. D. Hamilton, Chem. Rev. 2004, 104, 1201-1218.

[6] X. Liu, Top. Curr, Chem. 2005, 256, 1-37.

[7] a) M. M. Smith, W. Edwards, D. K. Smith, Chem. Sci. 2013, 4, 671-676; b) H. Bunzen, E. Kolehmainen, Molecules 2013, 18, 3745-3759; c) M. Suzuki, Y. Nakajima, M. Yumoto, M. Kimura, H. Shirai, K. Hanabusa, Org. Biomol. Chem. 2004, 2, 1155-1159; d) M. George, R. G. Weiss, J. Am. Chem. Soc. 2001, 123, 10393-10394.

[8] a) J. Shen, Y. Chen, J. Huang, J. Chen, C. Zhao, Y. Zheng, T. Yu, Y. Yang, H. Zhang, Soft Matter 2013, 9, 2017-2023; b) H. Svobodova, Nonappa, M. Lahtinen, Z. Wimmer, E. Kolehmainen, Soft Matter 2012, 8, 7840-7847; c) J. H. Lee, S. Kang, J. Y. Lee, J. H. Jung, Soft Matter 2012, 8, 6557-6563; d) M. M. Piepenbrock, N. Clarke, J. W. Steed, Soft Matter 2011, 7, 2412-2418; e) T. D. Hamilton, D. Buc• ar, J. Baltrusaitis, D. R. Flanagan, Y. Li, S. Ghorai, A. V. Tivanski, L. R. MacGillivray, J. Am. Chem. Soc. 2011, 133, 33653371; f) L. Yang, L. Luo, S. Zhang, X. Su, J. Lan, C. Chen, J. You, Chem. Commun. 2010, 46, 3938-3940.

[9] a) M. Segarra-Maset, V. J. Nebot, J. F. Miravet, B. Escuder, Chem. Soc. Rev 2013, DOI: $10.1039 / \mathrm{C} 2 \mathrm{CS} 35436 \mathrm{E}$; b) X. Yang, G. Zhang, D. Zhang, J. Mater. Chem. 2012, 22, 38-50.

[a] H. Bunzen, Dr. E. Kalenius, Prof. E. Kolehmainen Department of Chemistry, University of Jyväskylä P.O. Box 35, FIN-40014, University of Jyväskylä, Finland Fax: (+358) 142602501, E-mail: hana.svobodova@jyu.fi

[b] Dr. Nonappa

Department of Applied Physics, Aalto University School of Science P.O. Box 15100, FI-02150, Espoo, Finland

[c] Dr. Sami Hietala

Department of Chemistry, University of Helsinki

P.O. Box 55, FI-00014, Helsinki, Finland 


\section{Supporting information for}

\section{Subcomponent self-assembly: a quick way to novel metallogels}

Hana Bunzen, Nonappa, Elina Kalenius, Sami Hietala and Erkki Kolehmainen

\section{Synthesis}

\section{General}

Analytical grade reagents and solvents were used for the synthesis, purification and gelation studies. Cholesteryl chloroformate was purchased from Alfa Aesar, ethane-1,2diamine from J.T. Baker, and 2-pyridinecarboxaldehyde from Sigma Aldrich. 2-Pyridinecarboxaldehyde was freshly distilled before use. ${ }^{1} \mathrm{H}$ and ${ }^{13} \mathrm{C}$ NMR experiments were run with a Bruker Avance DRX 500 NMR spectrometer equipped with a direct observation BBO probehead working at $500.13 \mathrm{MHz}$ for ${ }^{1} \mathrm{H}$ and at $125.76 \mathrm{MHz}$ for ${ }^{13} \mathrm{C}$. NMR spectra were measured in $\mathrm{CDCl}_{3}$ and the chemical shifts were referenced to the solvent signal $\left(\delta=7.26 \mathrm{ppm}\right.$ for ${ }^{1} \mathrm{H}$, and $\delta=77.0 \mathrm{ppm}$ for ${ }^{13} \mathrm{C}$ from internal TMS). The numbering of the steroidal skeleton is according to the IUPAC rules. Molecular masses were measured either by using a Micromass LCT ESI-TOF mass spectrometer or by a VG AutoSpace 3500 HR-MS high resolution mass spectrometer. IR spectra were recorded on a Bruker Tensor 27 ATR FTIR spectrometer.

\section{Preparation of compound $\mathbf{1}$}

To a solution of ethane-1,2-diamine (2.54 mL, $47.011 \mathrm{mmol}, 20$ eq.) and dry triethylamine $\left(0.33 \mathrm{~mL}, 2.366 \mathrm{mmol}, 1\right.$ eq.) in dry dichloromethane $(30 \mathrm{~mL})$ at $0{ }^{\circ} \mathrm{C}$, a solution of cholesteryl chloroformate (1.00 g, $2.352 \mathrm{mmol}, 1 \mathrm{eq}$.) in dry dichloromethane $(30 \mathrm{~mL})$ was added dropwise. The mixture was stirred at ambient temperature under nitrogen atmosphere for $18 \mathrm{~h}$. The formed precipitate was filtrated off and the filtrate was washed four times with brine. The organic layer was dried over $\mathrm{Na}_{2} \mathrm{SO}_{4}$ and evaporated to dryness to give the desired product as a white solid in $96 \%$ yield $(1.072 \mathrm{~g}) . \delta_{\mathrm{H}}(500.13$ $\left.\mathrm{MHz} ; \mathrm{CDCl}_{3}\right): 0.68\left(3 \mathrm{H}, \mathrm{s}, 18-\mathrm{CH}_{3}\right), 0.86\left(6 \mathrm{H}, \mathrm{dd}, \mathrm{J}=2.2 ; 6.6 \mathrm{~Hz}, 26-\mathrm{CH}_{3}+27-\mathrm{CH}_{3}\right)$, $0.91\left(3 \mathrm{H}, \mathrm{d}, \mathrm{J}=6.6 \mathrm{~Hz}, 21-\mathrm{CH}_{3}\right), 1.01\left(3 \mathrm{H}, \mathrm{s}, 19-\mathrm{CH}_{3}\right), 2.81(2 \mathrm{H}, \mathrm{t}, \mathrm{J}=5.8 \mathrm{~Hz}$, ethylenediamine $), 3.21(2 \mathrm{H}, \mathrm{m}$, ethylenediamine $), 4.50(1 \mathrm{H}, \mathrm{m}, 3-\mathrm{CH}), 4.94(1 \mathrm{H}, \mathrm{bs}$, 
$\mathrm{NH}), 5.37(1 \mathrm{H}, \mathrm{m}, 6-\mathrm{CH}) . \delta_{\mathrm{C}}\left(125.7 \mathrm{MHz} ; \mathrm{CDCl}_{3}\right): 11.86(\mathrm{C}-18), 18.72(\mathrm{C}-21), 19.32(\mathrm{C}-$ 19), 21.06 (C-11), 22.54 (C-27), 22.79 (C-26), 23.84 (C-23), 24.29 (C-15), 28.00 (C-25), 28.19 (C-2), 28.22 (C-16), 31.90 (C-7), 31.90 (C-8), 35.79 (C-20), 36.20 (C-22), 36.58 (C-10), 37.02 (C-1), 38.59 (C-4), 39.53 (C-24), 39.77 (C-12), 41.83 (ethylenediamine), 42.33 (C-13), 43.74 (ethylenediamine), 50.06 (C-9), 56.18 (C-17), 56.72 (C-14), 74.34 (C-3), $122.46(\mathrm{C}-6), 139.87(\mathrm{C}-5), 156.41(\mathrm{C}=\mathrm{O}) ; v_{\max } / \mathrm{cm}^{-1}: 3337(\mathrm{NH}), 2937,2867$ $(\mathrm{CH}), 1715(\mathrm{C}=\mathrm{O},-\mathrm{O}), 1696(\mathrm{C}=\mathrm{O},-\mathrm{NH}), 1549(\mathrm{NH}$, bending $)$ and 1247s $(\mathrm{C}-\mathrm{O}) ; \mathrm{m} / \mathrm{z}$ $\left(\mathrm{ES}^{+}\right) 473.33\left([\mathrm{M}+\mathrm{H}]^{+}, 100 \%\right) ; \mathrm{m} / \mathrm{z}$ (HR-ESI) 473.4102, $\left[\mathrm{C}_{30} \mathrm{H}_{53} \mathrm{~N}_{2} \mathrm{O}_{2}+\mathrm{H}\right]^{+}$requires 473.4107.

\section{Preparation of metal complexes $\mathbf{3 a - c}$}

Compound 1, 2-pyridinecarboxaldehyde (2) and a metal salt, namely $\mathrm{Cu}\left(\mathrm{ClO}_{4}\right)_{2} \cdot 6 \mathrm{H}_{2} \mathrm{O}$, or $\mathrm{Ni}\left(\mathrm{ClO}_{4}\right)_{2} \cdot 6 \mathrm{H}_{2} \mathrm{O}$, or $\mathrm{Zn}\left(\mathrm{BF}_{4}\right)_{2} \cdot 6-7 \mathrm{H}_{2} \mathrm{O}$, were mixed in a molar ratio of 3:3:1 in a selected solvent. After mixing the components, a color change was observed - from colorless to a colored solution $\left(\mathrm{Cu}^{2+}\right.$ : blue-green, $\mathrm{Ni}^{2+}$ : yellow, $\mathrm{Zn}^{2+}$ : light yellow), indicating a complex formation.

\section{Gelation studies}

\section{Experimental details}

In a typical gelation test the calculated amounts of the components were mixed with the measured volume of the selected solvent in a sealed $5 \mathrm{~mL}$ test tube. The sample was shaken (or treated with ultrasound) and if needed also heated (if some of the components were insoluble in the selected solvent at ambient temperature), in order to prepare a clear solution. The resulting solution was allowed to stay without any disturbance and slowly cooled down to room temperature (if the sample was heated). Finally after 30-60 min, the test tubes were inverted to observe if the contents could still flow. Formation of a gel $(\mathrm{G})$, precipitate $(\mathrm{P})$, or solution $(\mathrm{S})$ was detected.

Minimum gelation concentration (MGC) was determined by scaling a minimum amount of gelator needed for a formation of stable gel. The tested concentrations were $5.0,4.0,3.0,2.0,1.5,1.0,0.75,0.5,0.25,0.10,0.08,0.07$, and $0.06 \%$ w/v. 
Photos of gels

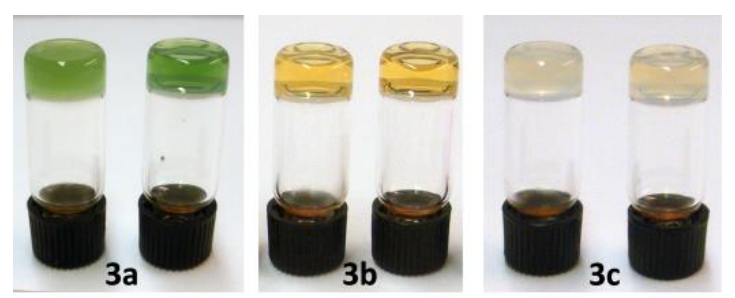

Figure S1. Gels of 3a-c formed in hexan-1-ol (2\% w/v, left) and octan-1-ol (2\% w/v, right).

\section{In situ gelation}
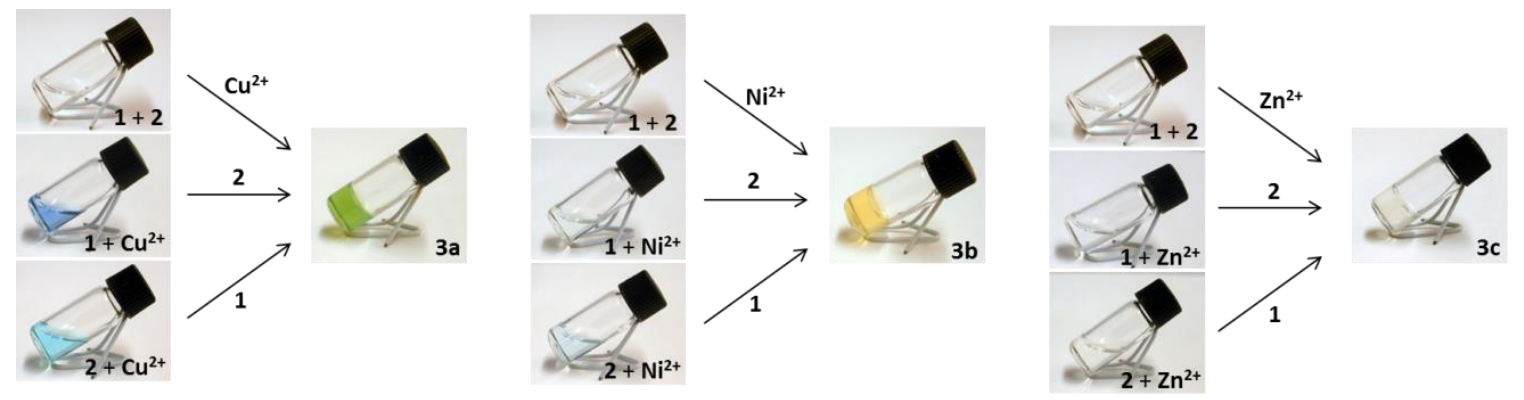

Scheme S1. In situ gelation of 3a-c in octan-1-ol (1\% w/v) at ambient temperature.

\section{Gel-to-sol phase transition temperature}

The gel-to-sol phase transition temperature $\left(\mathrm{T}_{\mathrm{g}}\right)$ was determined using an "inversion tube" method and the measurements were repeated three times for each sample. Gels of a constant volume were prepared to the sealed containers and stabilized overnight at $\mathrm{rt}$. After that they were placed into a water bath upside-down and gradually heated (1 ${ }^{\circ} \mathrm{C} / \mathrm{min}$ ). The temperature at which the gel fell under gravity was recorded as the gel-to-sol phase transition temperature $\left(\mathrm{T}_{\mathrm{g}}\right)$. 


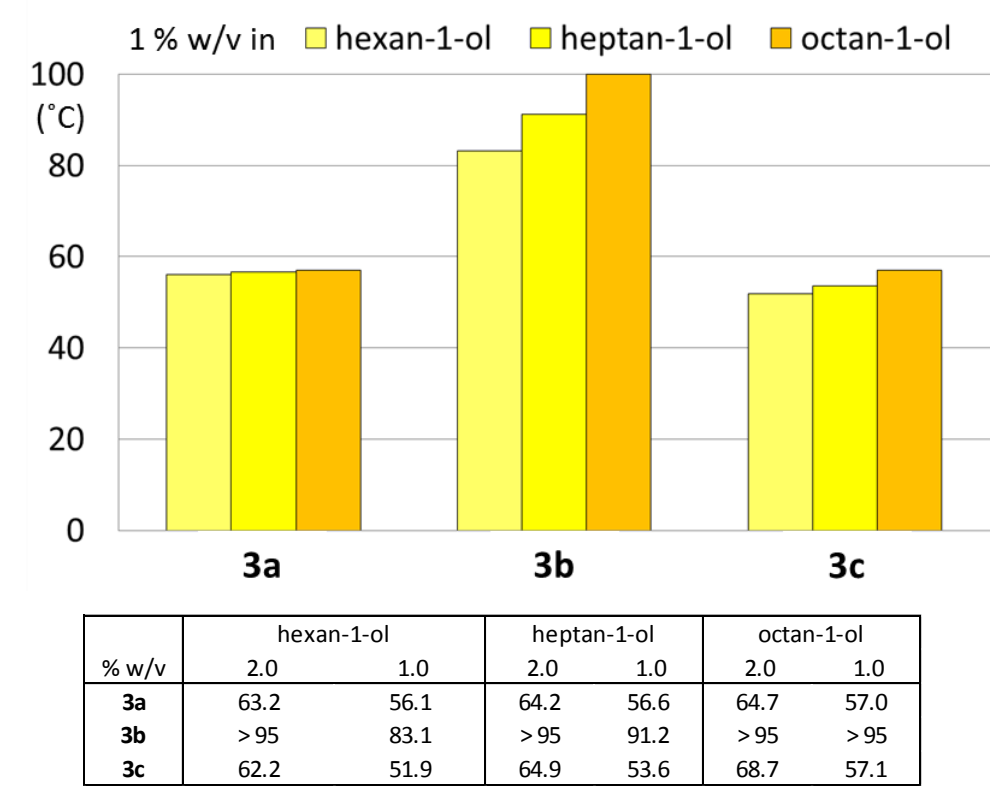

Figure S2. Gel-to-sol transition temperatures (in ${ }^{\circ} \mathrm{C}$ ) of gels of 3a-c in higher alcohols.

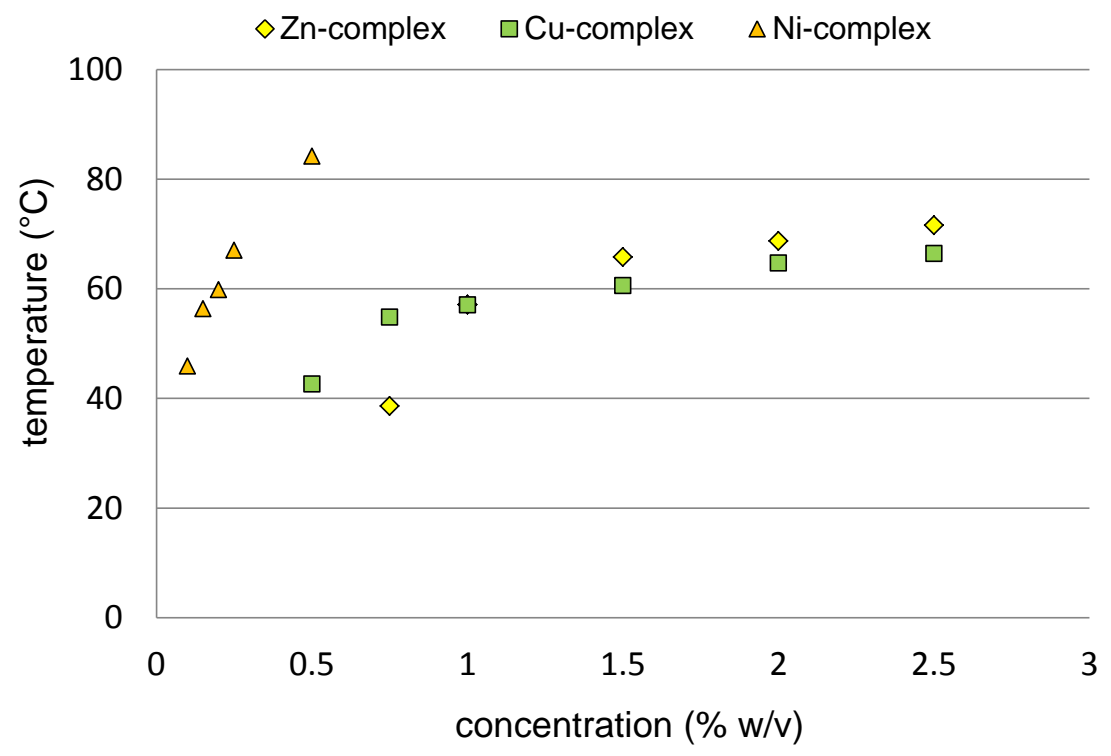

\begin{tabular}{|c|ccccccccccc|}
\hline$\% \mathrm{w} / \mathrm{v}$ & 2.5 & 2.0 & 1.5 & 1.0 & 0.75 & 0.5 & 0.25 & 0.20 & 0.15 & 0.10 & 0.05 \\
\hline 3a & 66.4 & 64.7 & 60.6 & 57.0 & 54.8 & 42.6 & - & - & - & - & - \\
3b & $>95$ & $>95$ & $>95$ & $>95$ & $>95$ & 84.1 & 67.0 & 59.8 & 56.3 & 45.9 \\
3c & 71.6 & 68.7 & 65.8 & 57.1 & 38.6 & - & - & - & - & - \\
\hline
\end{tabular}

Figure S3. Phase diagram of 3a-c in octan-1-ol. 
Different ligand-metal ratios and combinations of metal salts

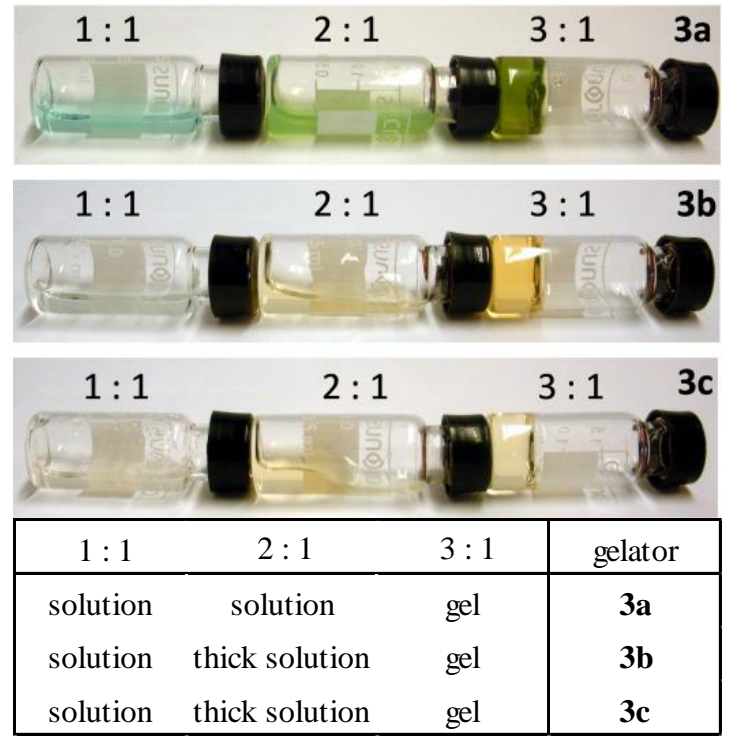

Figure S4. Gelators 3a-c in octan-1-ol (2\% w/v) at different ligand-metal ratio.

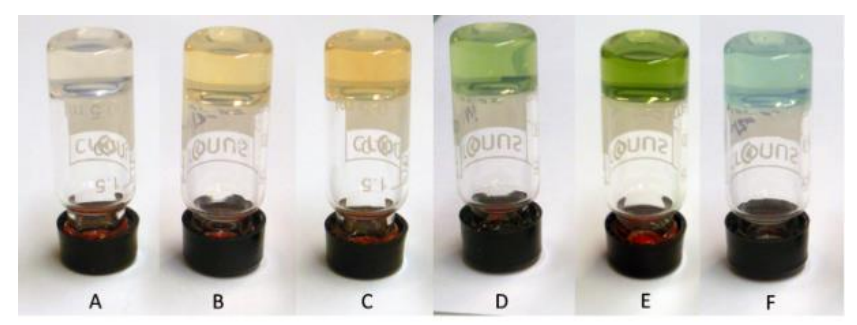

\begin{tabular}{|c|cccccc|}
\hline$\% \mathrm{w} / \mathrm{v}$ & $\mathrm{Zn}^{2+}$ & $\mathrm{Zn}^{2+} / \mathrm{Ni}^{2+}$ & $\mathrm{Ni}^{2+}$ & $\mathrm{Ni}^{2+} / \mathrm{Cu}^{2+}$ & $\mathrm{Cu}^{2+}$ & $\mathrm{Cu}^{2+} / \mathrm{Zn}^{2+}$ \\
\hline 2.0 & 68.7 & $>95$ & $>95$ & 90.6 & 64.7 & 68.3 \\
1.0 & 57.1 & 67.9 & $>95$ & 66.4 & 57.0 & 57.5 \\
\hline
\end{tabular}

Figure S5. Gels of metal complexes of a total ligand-metal ratio of 3:1 in octan-1-ol (1\% $\mathrm{w} / \mathrm{v}$ ) and their gel-to-sol transition temperatures (in $\left.{ }^{\circ} \mathrm{C}\right)$ : (A) ligand: $\mathrm{Zn}^{2+}(3: 1$, i.e. 3c), (B) ligand: $\mathrm{Ni}^{2+}: \mathrm{Zn}^{2+}(3: 0.5: 0.5),(\mathrm{C})$ ligand: $\mathrm{Ni}^{2+}\left(3: 1\right.$, i.e. 3b), (D) ligand: $\mathrm{Cu}^{2+}: \mathrm{Ni}^{2+}$ (3:0.5:0.5), (E) ligand: $\mathrm{Cu}^{2+}\left(3: 1\right.$, i.e. 3a), and (F) ligand: $\mathrm{Cu}^{2+}: \mathrm{Zn}^{2+}(3: 0.5: 0.5)$.
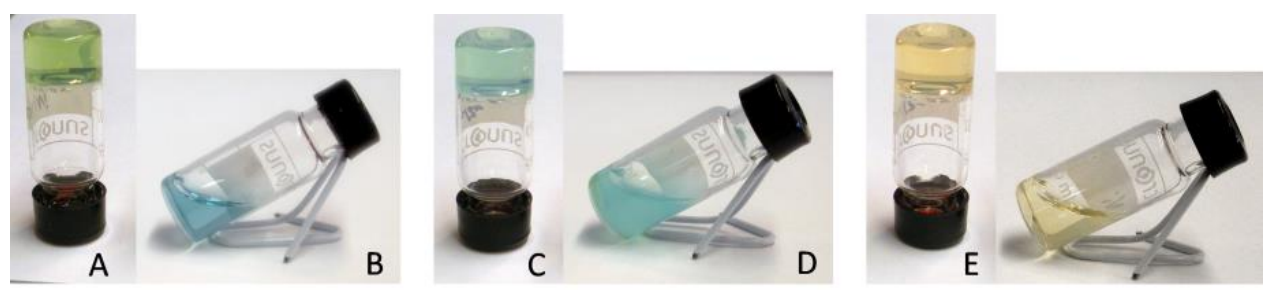

Figure S6. Metal complexes of different ligand-metal ratios in octan-1-ol (1\% w/v): (A) ligand: $\mathrm{Cu}^{2+}: \mathrm{Ni}^{2+}(3: 0.5: 0.5)$, (B) ligand: $\mathrm{Cu}^{2+}: \mathrm{Ni}^{2+}(3: 1: 1),(\mathrm{C})$ ligand: $\mathrm{Cu}^{2+}: \mathrm{Zn}^{2+}$ (3:0.5:0.5), (D) ligand: $\mathrm{Cu}^{2+}: \mathrm{Zn}^{2+}(3: 1: 1),(\mathrm{E})$ ligand: $\mathrm{Ni}^{2+}: \mathrm{Zn}^{2+}(3: 0.5: 0.5)$, and (F) ligand: $\mathrm{Ni}^{2+}: \mathrm{Zn}^{2+}(3: 1: 1)$. 
Stimuli-responsive gels

\section{EDTA experiments}

In the EDTA experiment, to a $1 \% \mathrm{w} / \mathrm{v}$ gel in octan-1-ol $(1 \mathrm{~mL})$, a saturated aqueous EDTA (sodium salt) solution $(1 \mathrm{~mL})$ was added. The sample was treated with ultrasound for 10-15 $\mathrm{min}$, and then was standing for $30 \mathrm{~min}$ without any disturbance. The formed phases were separated, and to the organic phase, new portions of aldehyde $\mathbf{2}$ and a metal salt $\left(\mathrm{Cu}^{2+}, \mathrm{Ni}^{2+}\right.$, or $\left.\mathrm{Zn}^{2+}\right)$ were added. A gel was reformed after intensive shaking of the sample at ambient temperature, or upon a heating/cooling cycle. The drawback of the experiment is the determination of the amount of aldehyde $\mathbf{2}$, which has to be added. Since the imine formation is a dynamic reversible reaction, a certain amount of $\mathbf{2}$ is presented in the sample in a free form and is washed out by the aqueous phase $\left(\log \mathrm{P}_{\mathrm{ow}}\right.$ partition coefficient for $n$-octanol/water system, of 2 is 0.714 ). Therefore, an additional amount of $\mathbf{2}$ has to be added. The exact amount depends on the intensity and duration of the extraction. However, it was found experimentally that an extra addition of $40-50 \%$ of $\mathbf{2}$ was in most cases sufficient and a small excess of $\mathbf{2}$ did not prevent a gel formation.

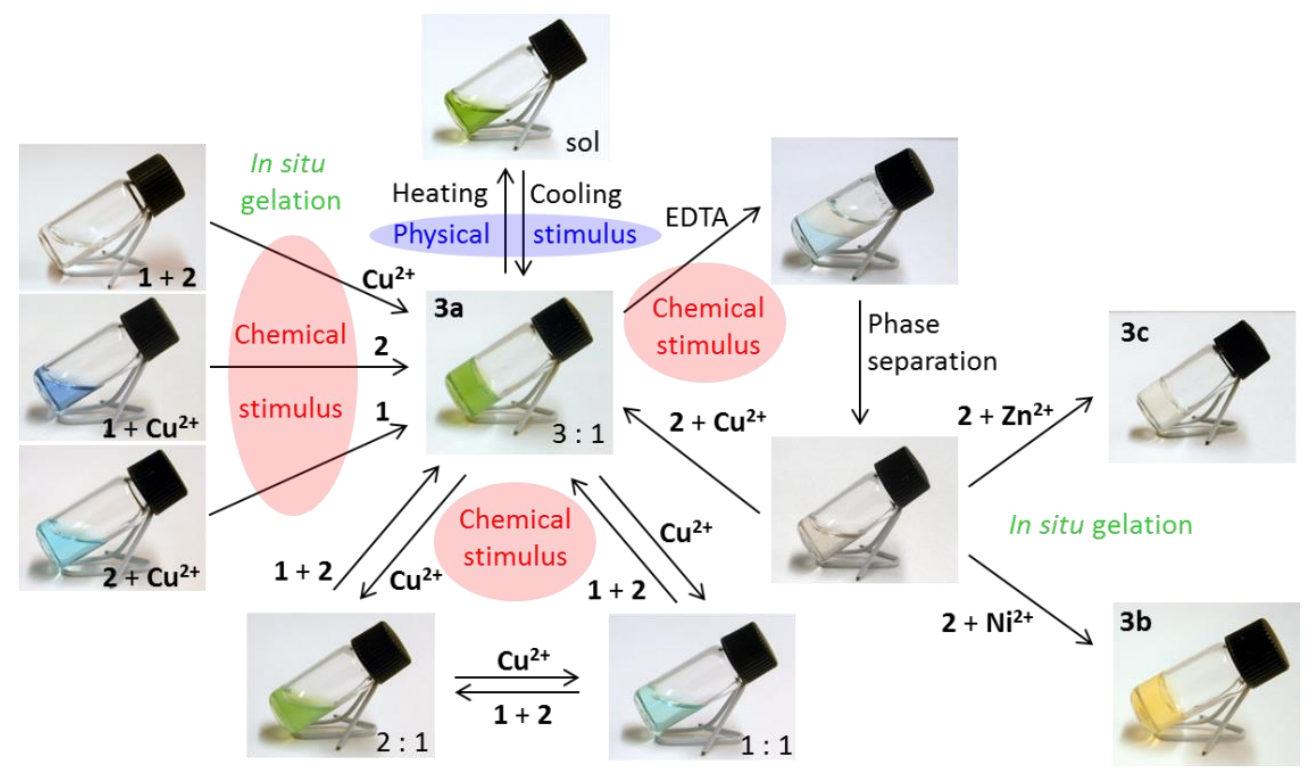

Scheme S2. Response of 3a in octan-1-ol (1\% w/v) to chemical and physical stimuli. 


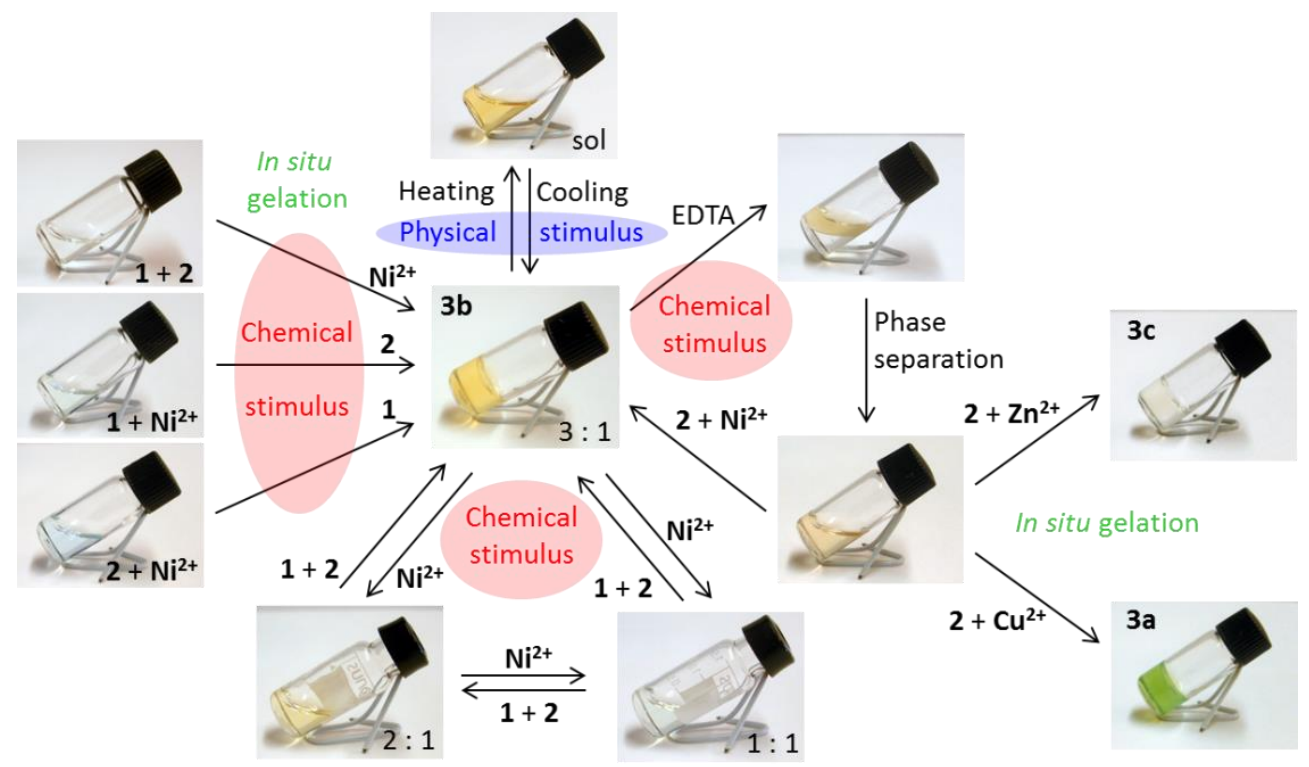

Scheme S3. Response of $\mathbf{3 b}$ in octan-1-ol (1\% w/v) to chemical and physical stimuli.

\section{Rheological measurements}

The rheological experiments were measured using a TA instruments AR2000 rheometer with $40 \mathrm{~mm}$ aluminium $2^{\circ}$ cone. The samples for the time sweep experiments were prepared by mixing the solutions of metal salts and the imine before the starting the measurements. Sol to gel transition was followed by an oscillatory time sweep from 20 min to $3 \mathrm{~h}$ depending on the gelation time of the components under investigation. The measurements were carried out within the linear viscoelastic regime (10\% strain) at 6.28 $\mathrm{rad} / \mathrm{s}$ frequency. After allowing the gel to attain sufficient stability (monitored by the time sweep experiment) frequency sweep and stress sweep experiments were performed. All the experiments were performed with controlled temperature of $20{ }^{\circ} \mathrm{C}$ with a Peltier heated plate. 


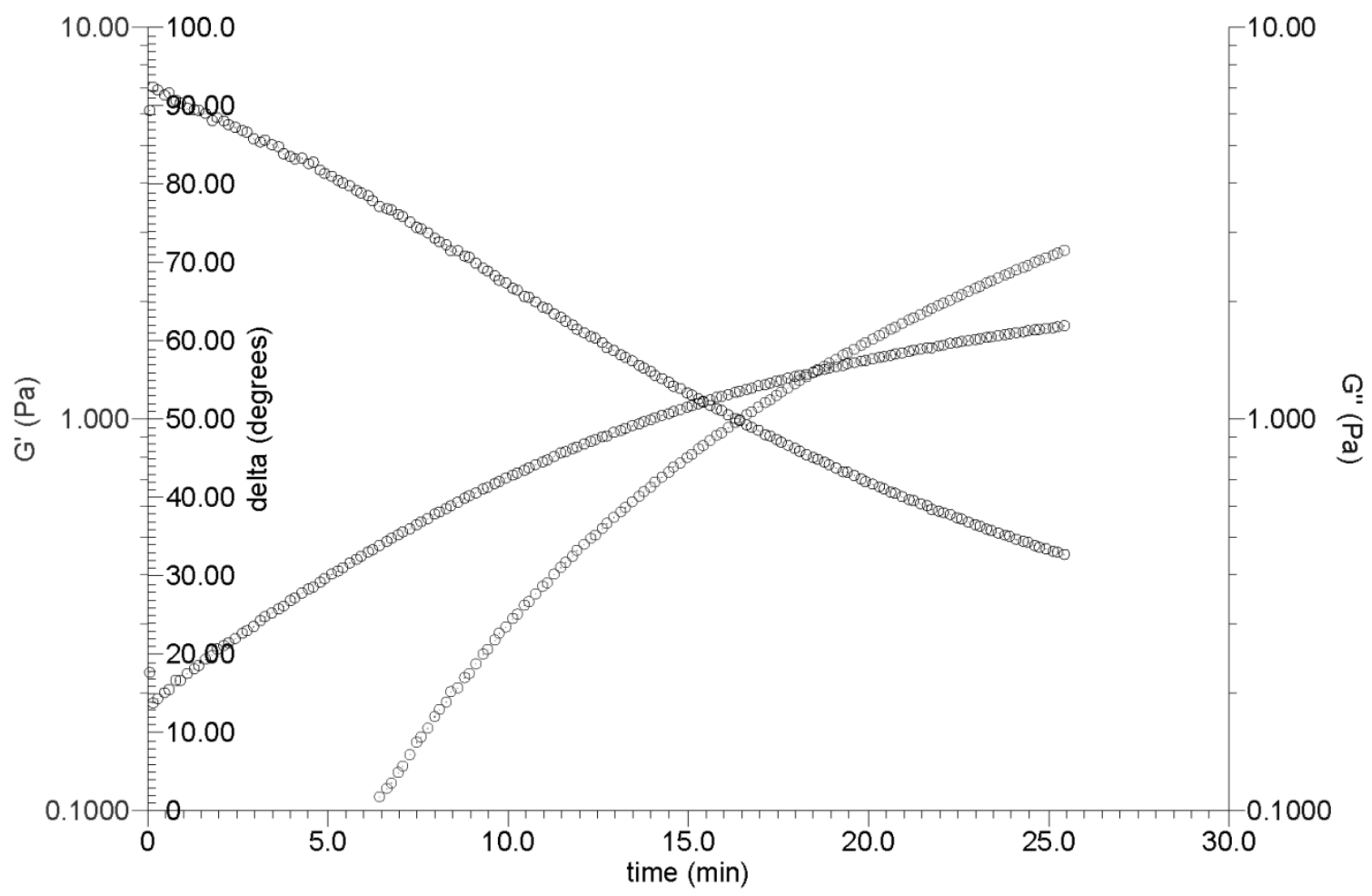

Figure S7. Time sweep experiment of gel of 3a formed in octan-1-ol (2\% w/v); $30 \mathrm{~min}$.

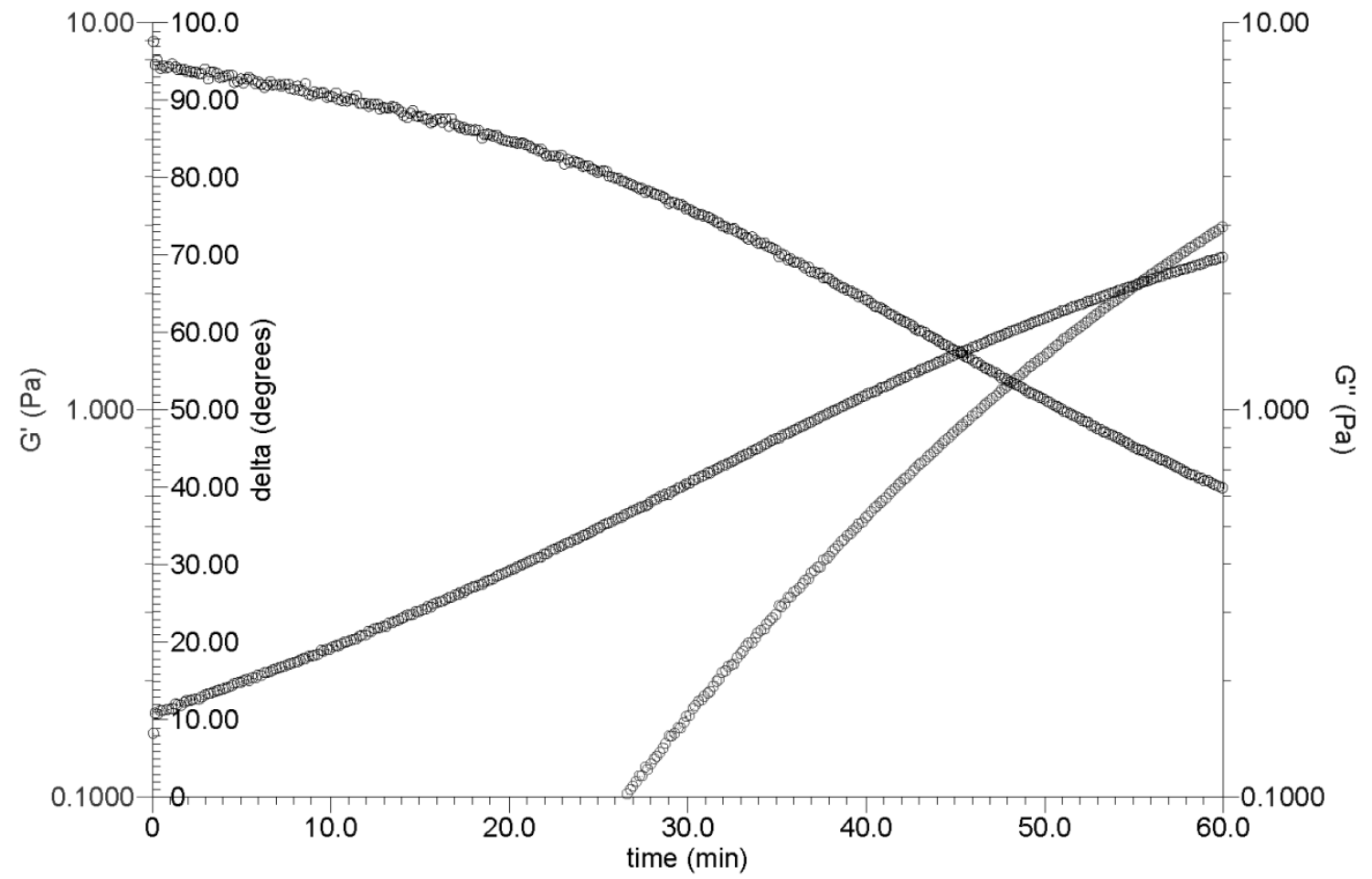

Figure S8. Time sweep experiment of gel of $\mathbf{3 b}$ formed in octan-1-ol (2\% w/v); $60 \mathrm{~min}$. 


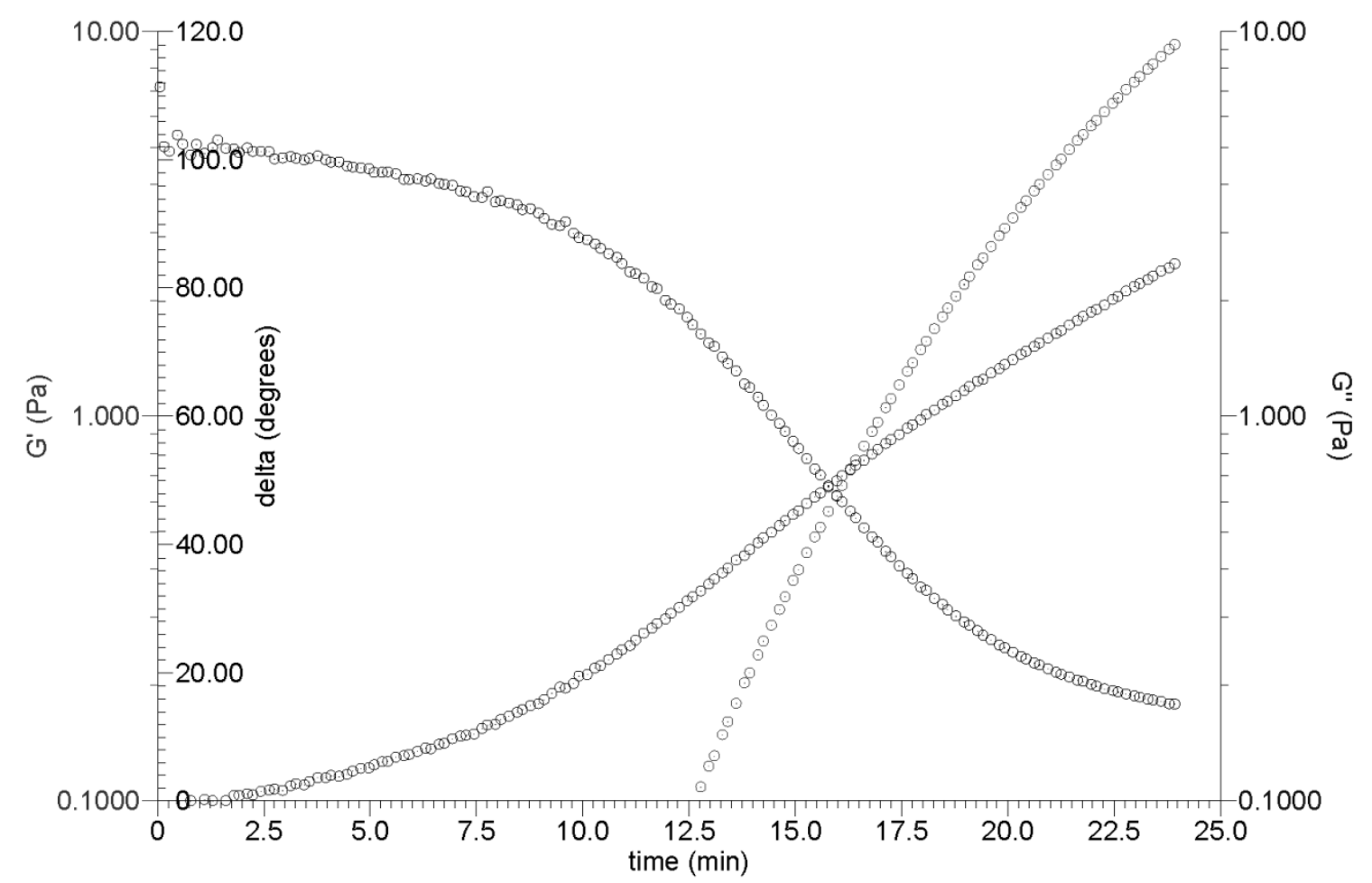

Figure S9. Time sweep experiment of gel of 3c formed in octan-1-ol (2\% w/v); $25 \mathrm{~min}$.

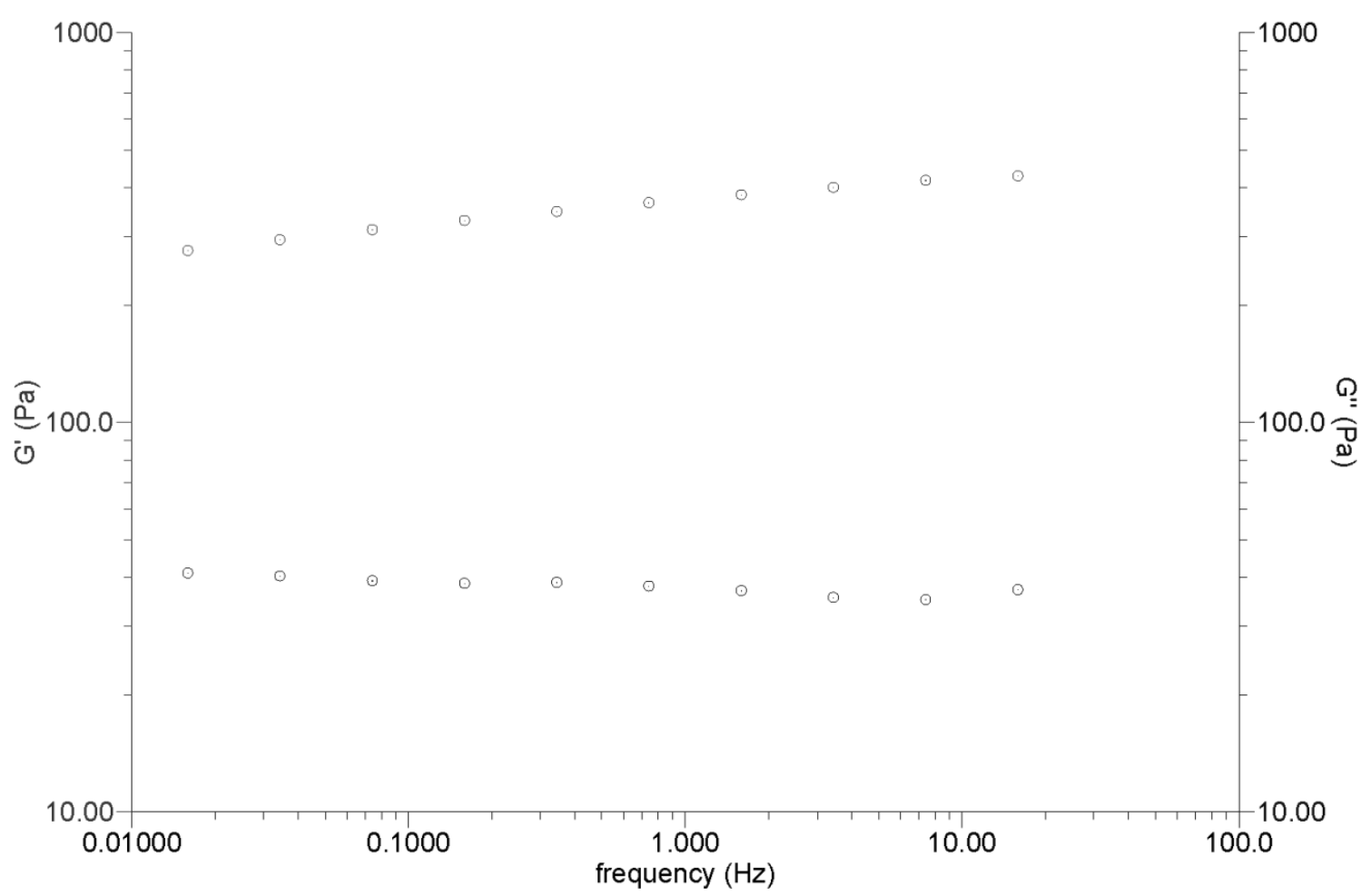

Figure S10. Frequency sweep experiment of gel of 3a formed in octan-1-ol (2\% w/v); measured after $3 \mathrm{~h}$ of the sample stabilization. 


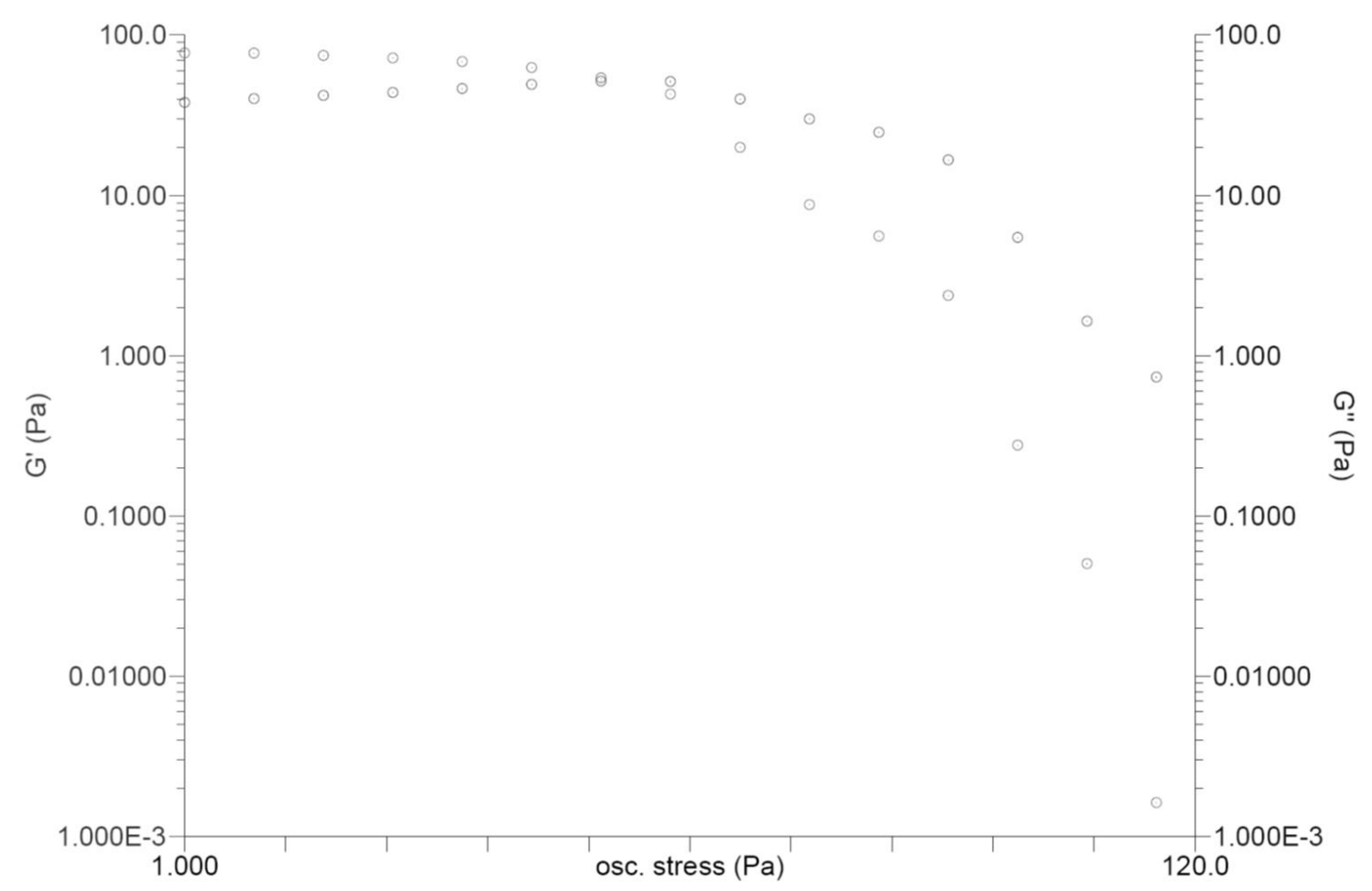

Figure S11. Stress sweep experiment of gel of $\mathbf{3 a}$ formed in octan-1-ol (2\% w/v).

\section{Speed of the gel formation}

In order to compare the speed of the gel formation of 3a-c, in situ gelation experiments were carried out for $2 \%$ and $1 \% \mathrm{w} / \mathrm{v}$ gels of 3a-c in octan-1-ol.

\section{a) Measured by time sweep experiments:}

After mixing the octan-1-ol solution of the amine (1) and aldehyde (2) with metal salts, oscillatory time sweep experiments were carried out from 20 min to $3 \mathrm{~h}$ depending on the gelation time of the system under investigation. The measurements were performed within the linear viscoelastic regime (10\% strain) at $6.28 \mathrm{rad} / \mathrm{s}$ frequency and the results are summarized in Figure S12. 


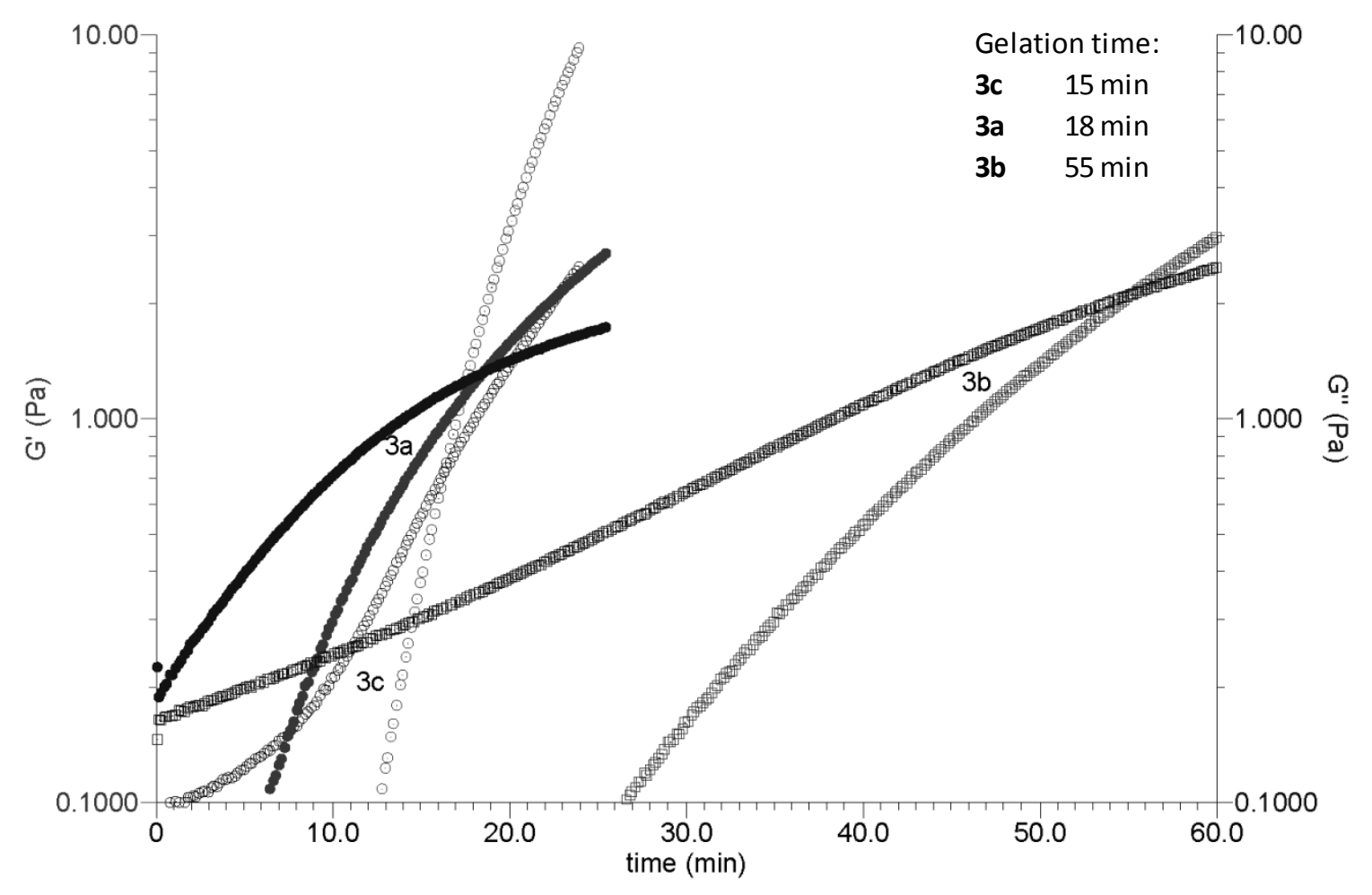

Figure S12. Time sweep experiments of gels of 3a-c formed in octan-1-ol (2\% w/v).

\section{b) Measured upon ultrasonic treatment:}

After mixing the beforehand dissolved components, the samples were treated with ultrasound for $90 \mathrm{~s}$. After that they were carefully checked by an inverted test tube method. The time, when the test tube could be turned up-side-down with the immobilized gel, was determined as the gelation time (Table S1).

Table S1. Time needed for an effective gel formation of 3a-c in octan-1-ol at different concentrations.

\begin{tabular}{|c|cc|}
\hline & \multicolumn{2}{|c|}{ Gels in octan-1-ol } \\
$\% \mathrm{w} / \mathrm{v}$ & 2.0 & 1.0 \\
\hline $3 \mathbf{c}$ & $50 \mathrm{~s}$ & $6.5 \mathrm{~min}$ \\
$3 \mathbf{3 a}$ & $90 \mathrm{~s}$ & $9 \mathrm{~min}$ \\
3b & $10 \mathrm{~min}$ & $24 \mathrm{~min}$ \\
\hline
\end{tabular}




\section{Mass spectrometry measurements}

The mass spectrometric experiments were performed with a QSTAR Elite ESI-Q-TOF mass spectrometer equipped with an API 200 TurbolonSpray ESI source from AB Sciex (former MDS Sciex) in Concord, Ontario (Canada). The gels of 3a-c in octan-1-ol (1\% w/v) were first dissolved in $\mathrm{CHCl}_{3}$ and then diluted in $\mathrm{CH}_{3} \mathrm{CN}$ to obtain $20 \cdot 10^{-6} \mathrm{M}$ samples for measurements. The samples were injected into the ESI source with a flow rate of $5 \mu \mathrm{L} / \mathrm{min}$. Room-temperature nitrogen was used as nebulization (30 psi) and as curtain gas (18 psi). The ion-source voltages were $4.8 \mathrm{kV}$ for capillary, $20 \mathrm{~V}$ for the orifice plate (declustering potential), $20 \mathrm{~V}$ as potential difference between skimmer and pre-quadrupole, and $250 \mathrm{~V}$ for the potential difference between the focusing ring and prequadrupole. Accumulation delay of 1s, ion release delay of $6 \mathrm{~ms}$ and ion release width of $5 \mathrm{~ms}$ were used. Each spectrum was an average of spectra collected within 2 to $5 \mathrm{~min}$, each of these containing 20 individual scans that were averaged before being sent from the instrument to data system. The measurement and data handling was accomplished with Analyst ${ }^{\circledR}$ QS 2.0 Software. Mass spectra were externally calibrated using sodium trifluoroacetate. The composition of the ions was verified by CID measurements as well as by comparison between experimental and theoretical mass values and isotopic distributions. 
A

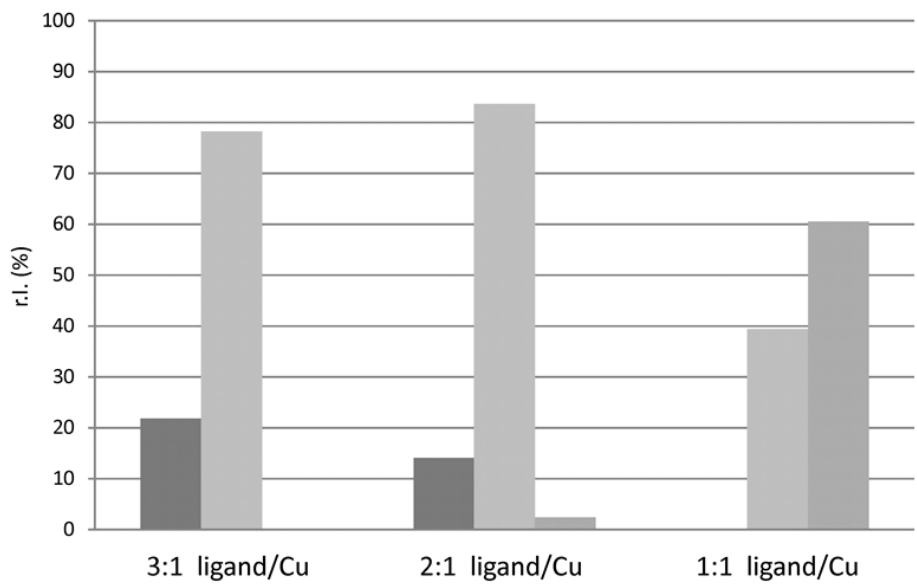

घ:1 complexes

2:1 complexes

1:1 complexes

B

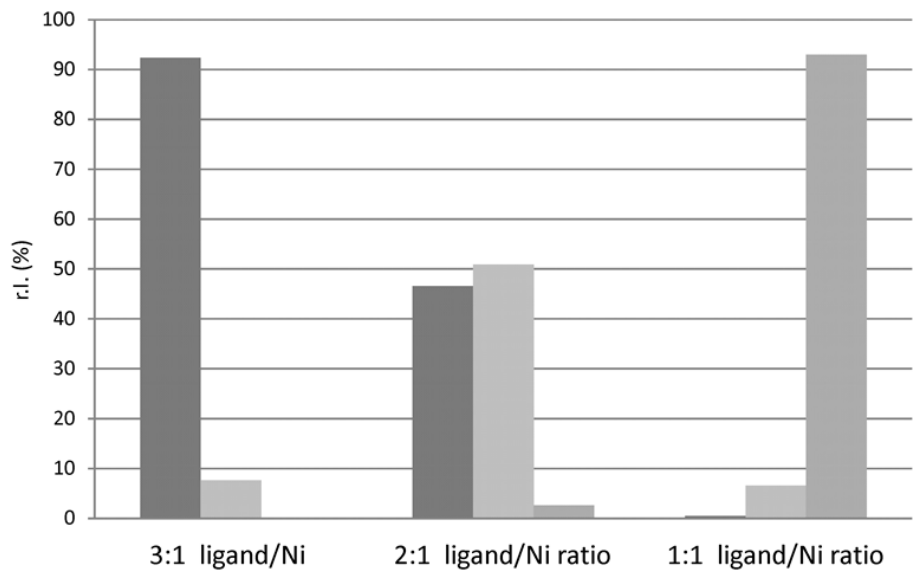

- 3:1 complexes

2:1 complexes

1:1 complexes

C

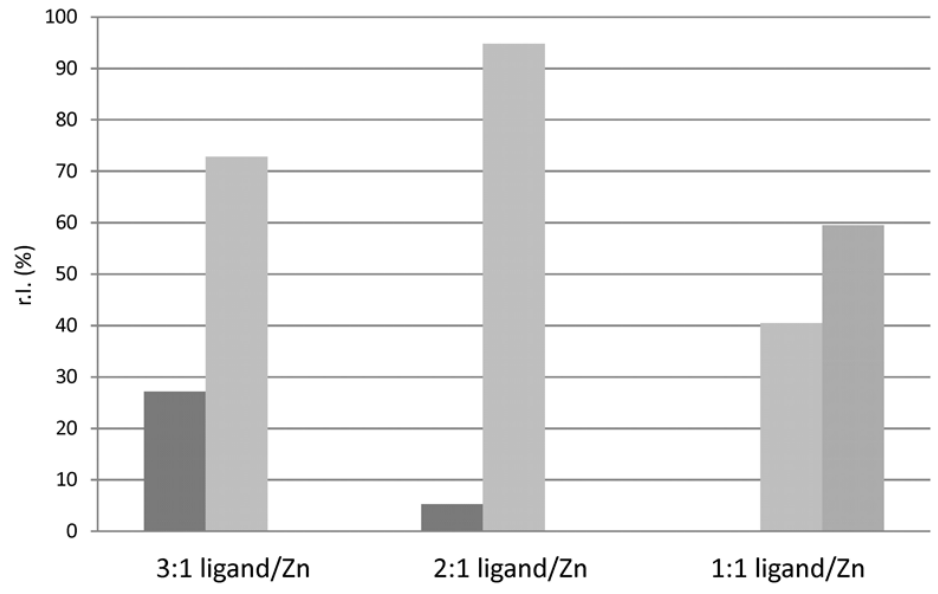

\section{[ 3:1 complexes}

2:1 complexes

—1:1 complexes

Figure S13. Relative intensities of 3:1, 2:1 and 1:1 complexes observed in ESI-Q-TOF mass spectra measured from $\mathrm{Cu}^{2+}(\mathrm{A}), \mathrm{Ni}^{2+}(\mathrm{B})$ and $\mathrm{Zn}^{2+}(\mathrm{C})$ gel samples with the ligand/metal ratios of $3: 1,2: 1$ and $1: 1$. 

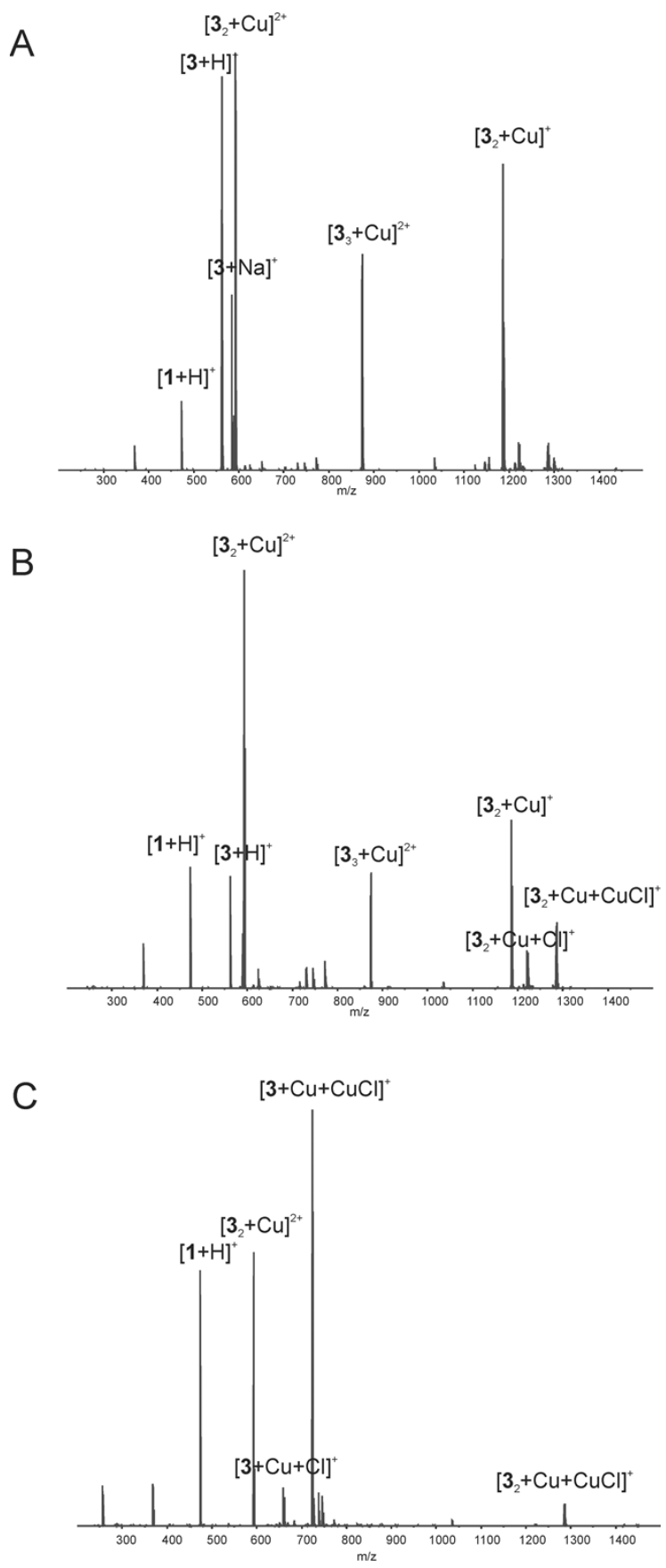

Figure S14. ESI-Q-TOF mass spectra measured from gel samples with the ligand-Cu ratio of 3:1 (A), 2:1 (B) and 1:1 (C). 
A

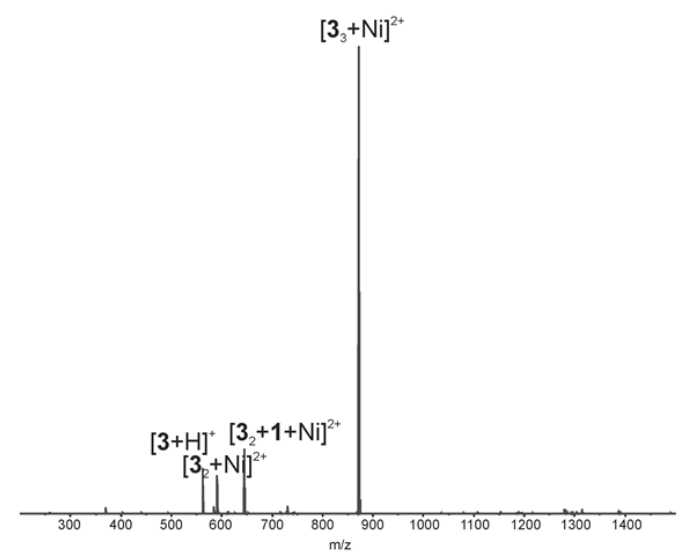

B

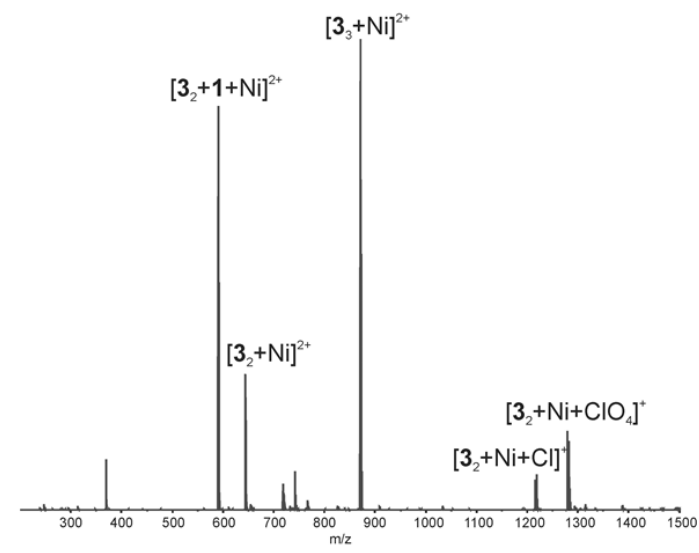

C

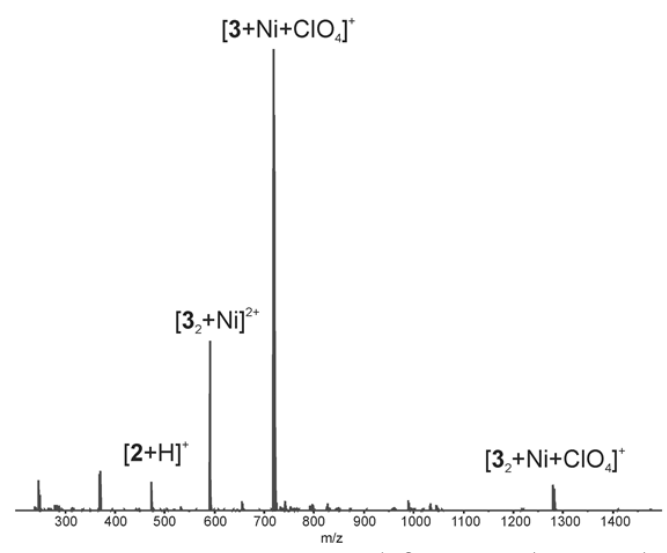

Figure S15. ESI-Q-TOF mass spectra measured from gel samples with the ligand-Ni ratio of 3:1 (A), 2:1 (B) and 1:1 (C). 

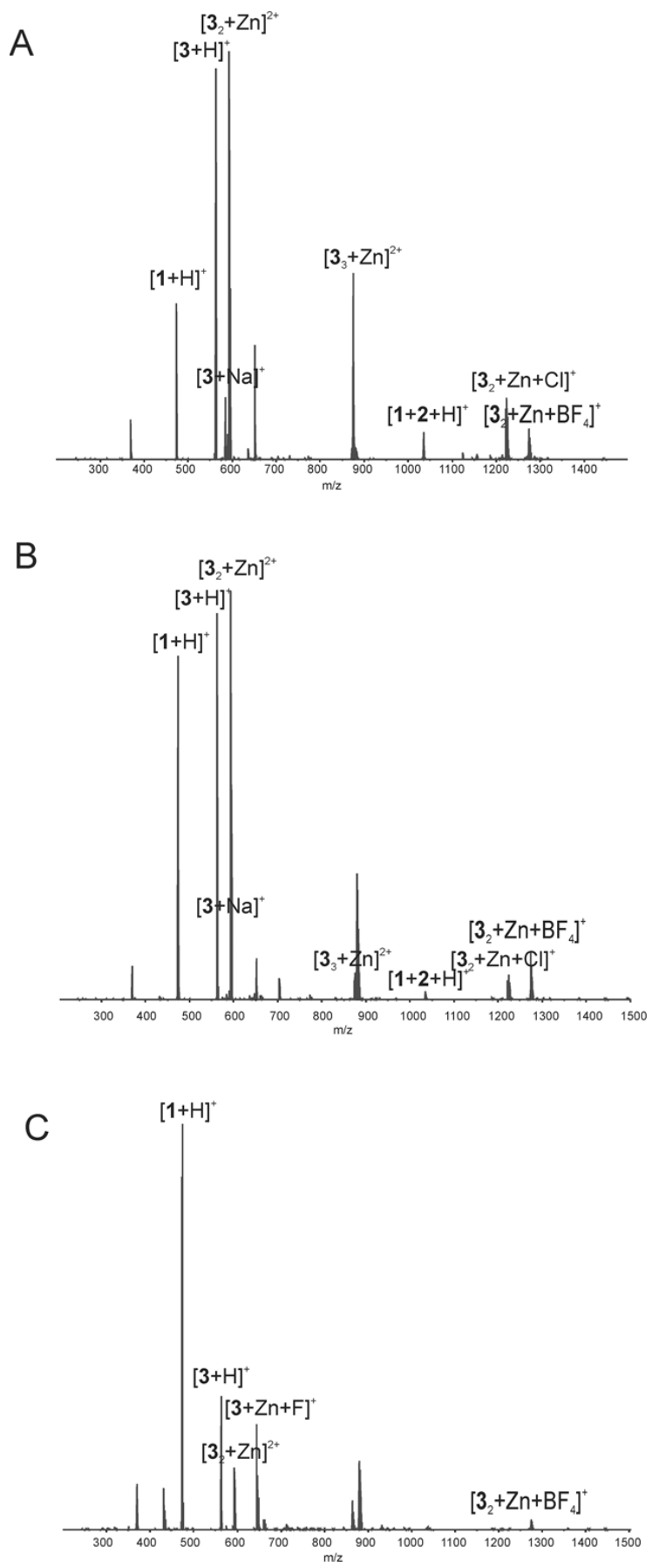

Figure S16. ESI-Q-TOF mass spectra measured from gel samples with the ligand-Zn ratio of $3: 1(\mathrm{~A}), 2: 1(\mathrm{~B})$ and $1: 1(\mathrm{C})$. 


\section{TEM measurements}

Transition electron micrographs of xerogels of 3a-c were acquired using a Technai 12 transmission electron microscope (FEI, Hillsboro, OR, USA) operated at $120 \mathrm{kV}$ and equipped with a Gatan Ultrascan 1000 CCD camera (Gatan Inc., Pleasanton, CA, USA). The samples for TEM measurements were prepared by placing $5 \mu \mathrm{L}$ of a gel solution (1.0 $\%$ w/v octan-1-ol gel dissolved by heating) on a TEM grid (carbon film only 200 mesh on gold). For dilute samples $(0.1 \% \mathrm{w} / \mathrm{v}), 100 \mu \mathrm{L}$ of the $1 \% \mathrm{w} / \mathrm{v}$ gel was diluted by octan-1ol to $1.0 \mathrm{~mL}$. The samples were allowed to dry at ambient conditions for $24 \mathrm{~h}$.

A)
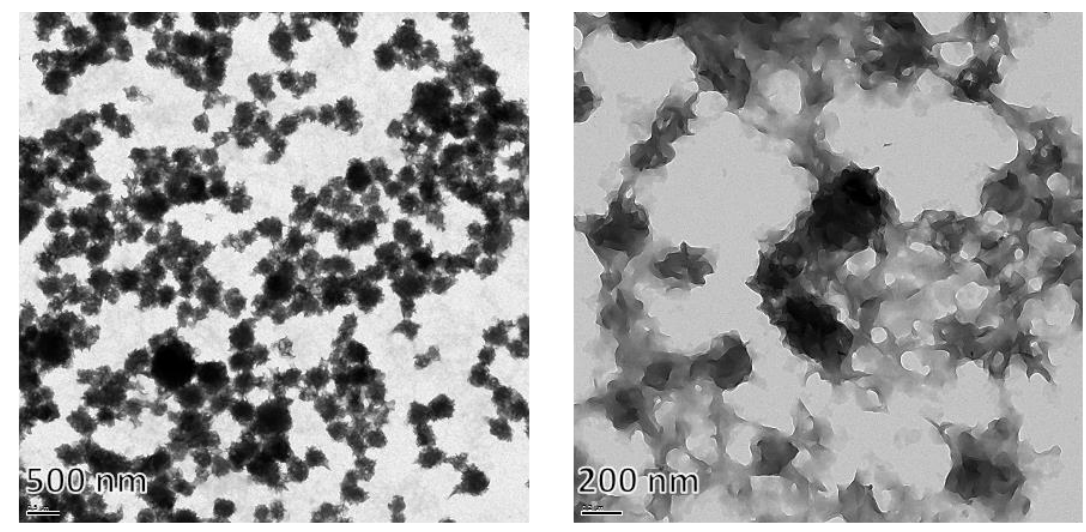

B)
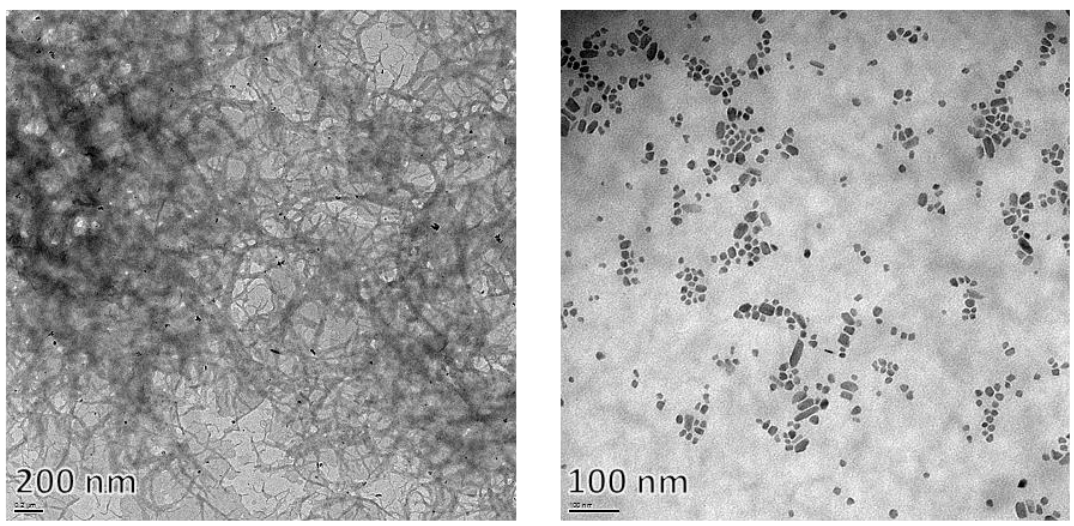

C)
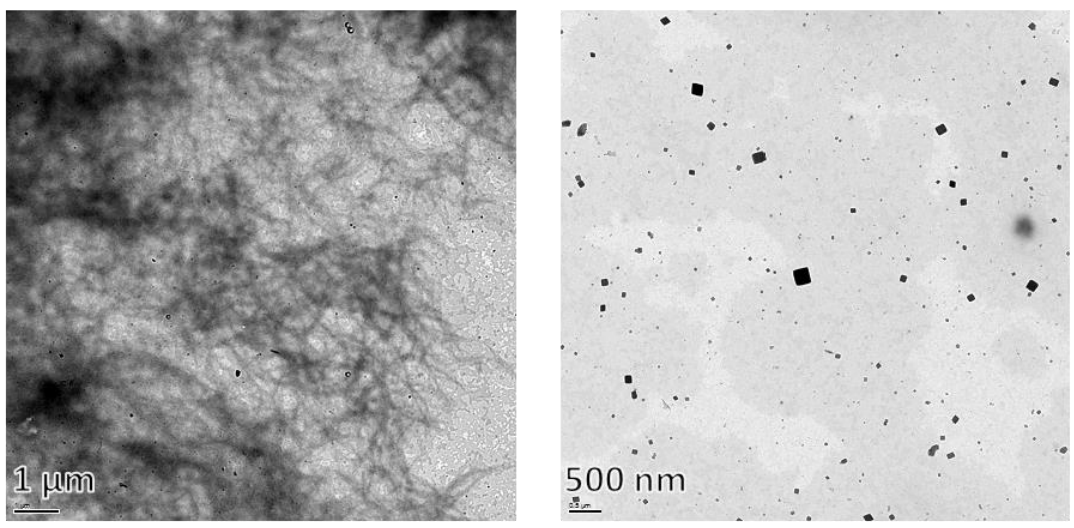

Figure S17. TEM micrographs of xerogels obtained from gels in octan-1-ol $(1.0 \% \mathrm{w} / \mathrm{v})$ of $\mathbf{3 a}(\mathrm{A}), \mathbf{3 b}(\mathrm{B})$, and $\mathbf{3 c}(\mathrm{C})$ revealing a fibrillar network and nanoparticle formation. 